\title{
Passivity-Based Switching Control for Stabilization of Wheeled Mobile Robots
}

\author{
Dongjun Lee \\ Department of Mechanical, Aerospace and Biomedical Engineering \\ University of Tennessee - Knoxville \\ 502 Dougherty Hall, 1512 Middle Dr. \\ Knoxville, TN 37996 USA \\ E-mail: djlee@utk.edu
}

\begin{abstract}
We propose a novel switching control law for the posture stabilization of a wheeled mobile robot, that utilizes the (energetic) passivity of the system's open-loop dynamics with nonnegligible inertial effects. The proposed passivity-based switching control law ensures that the robot's $(x, y)$-position enters into an arbitrarily small (as specified by user-designed error-bound) level set of a certain navigation potential function defined on the $(x, y)$ plane, and that its orientation converges to a target angle. Under this passivity-based switching control, the robot moves back and forth between two submanifolds in such a way that the navigation potential is strictly decreasing during this inter-switching move. Once the system's $(x, y)$-trajectory enters such a desired level set, at most one more switching occurs to correct orientation. Simulation is performed to validate/highlight properties of the presented control law.
\end{abstract}

\section{INTRODUCTION}

Wheeled mobile robots define one of the most important classes of robotic systems in practice. Let alone the ubiquitous automobiles, we can find them in such a variety of practical applications as material handling (e.g. Cobot [1]), space exploration (e.g. NASA Rover [2]), smart wheelchairs (e.g. NavChair [3]), and, recently, mobile sensor networks (e.g. [4]).

In addition to its practical importance, this wheeled mobile robot also constitutes a theoretically rich dynamical system due to the presence of nonholonomic constraints (i.e. noslip condition of wheels). These constraints only restrict the admissible velocity space but not that of the configuration [5]. Because of these nonholonomic constraints, control design and analysis become substantially more involved. For instance, as shown in the celebrated work [6], any continuous timeinvariant state feedback, which would work just fine if there is no nonholonomic constraints, now can not stabilize the position and orientation of the wheeled mobile robot at the same time (i.e. posture stabilization problem).

On the other hand, (energetic) passivity of open-loop robotic systems (i.e. passive with kinetic energy and mechanical power as storage function and supply rate [7]) has been a very powerful concept in many control problems in robotics: general motion control problem including adaptive and robust controls [8], teleoperation and haptic interface [9], [10], biped walking robot [11], and multirobot cooperative control [12], [13], to name a few. This is one of the most fundamental properties of robotic systems, holding for any choice of coordinate systems (e.g. euler-angle or quaternion for $S O(3)$ ). However, so far, it has been largely overlooked for the control problem of wheeled mobile robots and nonholonomic mechanical systems in general.

In this paper, we aim to bring this fundamental passivity property $^{1}$ into the posture stabilization problem of a wheeled mobile robot with second-order Lagrangian dynamics. The outcome is a novel switching control law, which, by utilizing the passivity property and energetic structure of the wheeled mobile robot, can ensure that the robot's orientation converges to a target value, while driving the robot's $(x, y)$-trajectory to a desired position on the plane within a user-specified errorbound.

The main idea of our control design can be roughly summarized as follows. Consider a wheeled mobile robot in Fig, 1. We first decouple its $(x, y)$-dynamics and orientation dynamics from each other by a certain feedback. Then, on the $(x, y)$ plane, we design a navigation potential [14], which may also incorporate other control objectives on the top of the posture stabilization (e.g. obstacle avoidance). With this potential, we switch the orientation angle between $\theta_{1}$ and $\theta_{2}$ (with some damping control) in such a way that the wheeled mobile robot will move between two one-dimensional submanifolds (each specified by $\theta_{1}$ and $\theta_{2}$ ) while guaranteeing that the navigation potential is strictly decreasing between the switchings. Thus, with these switchings, the system's $(x, y)$-position moves toward the minimum of the navigation potential. Once the system's $(x, y)$-position is close enough (as specified by a user-designed error-bound) to this minimum, at most one more switching occurs for correction of orientation while keeping the system's position still close to the minimum as specified by the error-bound.

Numerous feedback control methods have been proposed for the posture stabilization of wheeled mobile robots (e.g. [15], [16], [17], [18], [19], [20], [21]). However, to our best knowledge, none of them (other than one exception of

\footnotetext{
${ }^{1}$ Here, we utilize this passivity property mainly as an intrinsic structuralproperty of the (open-loop) system relating mechanical power and energy. This passivity may also be related to the energy efficiency of the controller system (e.g. biped walking), although this is not the direction taken in this paper.
} 


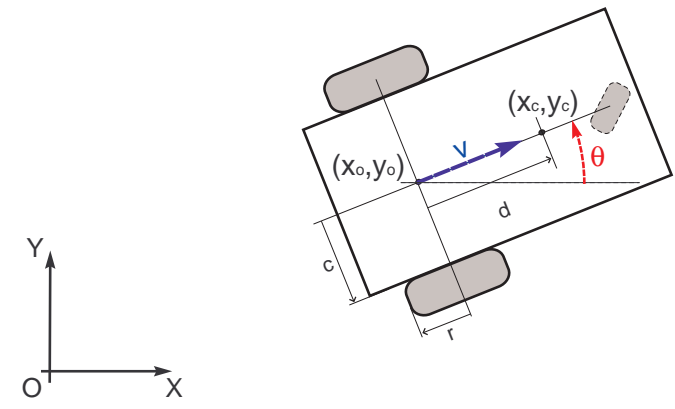

Fig. 1. Wheeled mobile robot with the center-of-mass at $\left(x_{c}, y_{c}\right)$ and the geometric center at $\left(x_{o}, y_{o}\right)$, both w.r.t. the reference frame $(O, X, Y)$.

[22]) exploits this intrinsic passivity property of (open-loop) wheeled mobile robots. In [22], the authors proposed a control law, which stands upon the port-controlled Hamiltonian structure of the nonholonomic system and a time-varying canonical transformation. This transformation preserves the open-loop passivity, which is central to enure their stabilization. However, a nontrivial partial differential equation needs to be solved there to find this transformation. Moreover, convergence of their obtained control law is very slow, as often found to be so with time-varying controls (e.g. [16]). Some switching control laws for the posture stabilization of wheeled mobile robots have also been proposed. However, to our best knowledge, all of them (e.g. [18], [19], [20]) are derived for the first-order kinematic model of wheeled mobile robots, thus, it is not clear how (of if) we can use them in many practical applications where the actual control inputs are torques and/or the inertial effect of the robot is not negligible.

Our passivity-based switching control relies on intrinsic energetic/geometric concepts and entities (e.g. passivity, submanifolds, dissipation, decoupling), which are not necessarily limited to the wheeled mobile robot and its posture stabilization problem. Rather, we think our passivity-based switching control idea must be applicable to more general control problems of possibly more general mechanical systems with nonholonomic constraints as well. As an example of such extensions, in this paper, we briefly present a preliminary result of an application of this passivity-based switching idea to the coordination problem of multiple wheeled mobile robots (with no motion planning). More detailed exposition of this and other extensions will be reported in future publications.

The rest of this paper is organized as follows. Dynamics of the wheeled mobile robot and its passivity property will be derived in Sec. II, and the passivity-based switching control law will be presented and analyzed in Sec. III. Simulation results to validate/highlight properties of the proposed control law will be given in Sec. IV. A preliminary result of extension to the multiple wheeled mobile robots will be presented in Sec. V, and summary and some concluding remarks on future research will be made in Sec. VI.

\section{WheELED Mobile Robot}

In this section, we derive the dynamics of the wheeled mobile robot projected on the admissible distribution (i.e. along the direction of the velocity, which does not violate the nonholonomic constraints). For more details, refer to [5].

Consider a 3-degree of freedom (DOF) wheeled mobile robot with the configuration $q=[x, y, \theta] \in S E(2)$, where $(x, y):=\left(x_{o}, y_{o}\right)$ is the position of the geometric center and $\theta \in S$ is the orientation of the robot w.r.t. the inertial frame $(O, X, Y)$. See Fig. 1. Here, we assume that the wheeled mobile robot has two independently-controlled rear wheels, and one front passive castor to avoid tipping over of the robot. We also assume that their inertias are negligible.

Then, the nonholonomic constraints (i.e. no slip condition) can be written by $A(\theta) \dot{q}=0$ with

$$
A(\theta):=\left[\begin{array}{lll}
\sin \theta & -\cos \theta & 0
\end{array}\right] \in \Re^{1 \times 3}
$$

and the admissible velocity (i.e. not violating the nonholonomic constraints) of the wheeled mobile robot is given by

$$
\dot{q}=S(q)\left(\begin{array}{c}
v \\
w
\end{array}\right), \quad S(q):=\left[\begin{array}{cc}
\cos \theta & 0 \\
\sin \theta & 0 \\
0 & 1
\end{array}\right]
$$

where $v \in \Re$ is the forward-velocity of the robot's geometric center and $w=\dot{\theta}$ (see Fig. 1). Here, the column vectors of $S(q) \in \Re^{3 \times 2}$ constitute the basis of the admissible velocity space at $q$, i.e., $\Delta_{q}:=\left\{\dot{q} \in \Re^{3} \mid A(q) \dot{q}=0\right\}$. This $\Delta_{q}$ is a 2 -dimensional linear vector space. By collecting this $\Delta_{q}$ over all $q$, we can get the (regular) admissible distribution $\Delta$.

Then, the dynamics of the wheeled mobile robot projected on $\Delta$ can be written by

$$
D(\theta) \dot{\nu}+Q(\theta, \dot{\theta}) \nu=u
$$

with $\nu:=[v, w]^{T}, u=\left[u_{v}, u_{w}\right]^{T}$,

$$
D:=\left[\begin{array}{cc}
m & 0 \\
0 & I
\end{array}\right], \quad Q:=\left[\begin{array}{cc}
0 & -m d \dot{\theta} \\
m d \dot{\theta} & 0
\end{array}\right]
$$

where $d \geq 0$ the distance between $\left(x_{c}, y_{c}\right)$ and $\left(x_{o}, y_{o}\right), m$ is the mass of the robot, and $I:=I_{c}+m d^{2}$ is the moment of inertia of the robot w.r.t. $\left(x_{o}, y_{o}\right)$ with $I_{c}$ being that of the robot w.r.t. $\left(x_{c}, y_{c}\right)$. Also, $u_{v}=\frac{1}{r}\left(\tau_{r}+\tau_{l}\right)$ and $u_{w}=\frac{c}{r}\left(\tau_{r}-\tau_{l}\right)$, where $\tau_{r}, \tau_{l} \in \Re$ are the torques of the right and left rear wheels, $r>0$ is the radius of the wheels, and $c>0$ is the half of the cart width. See Fig. 1. Since this is the dynamics projected on the admissible distribution $\Delta$, no constraint force (i.e. terms with Lagrange multiplier [5]) shows up here.

Using the skew-symmetricity of $\dot{D}-2 Q$, we can show that this wheeled mobile robot possesses the (energetic) passivity property [10]:

$$
\frac{d}{d t} \kappa=u^{T} \nu
$$

where $\kappa:=\frac{1}{2} m\left(\dot{x}^{2}+\dot{y}^{2}\right)+\frac{1}{2} I w^{2}=\frac{1}{2} m v^{2}+\frac{1}{2} I w^{2}$ is the total kinetic energy. Here, $\kappa$ and $u^{T} \nu$ serve as the storage function and the supply rate of the standard passivity definition [7]. 
Note that the dynamics of $v$ and $w$ in (2) are coupled with each other via the Coriolis terms $Q \nu$. Since the Coriolis matrix $Q$ is skew-symmetric, this coupling is energetically passive, i.e. does not generate nor dissipate energy. This can be shown s.t.: with $\kappa_{v}:=\frac{1}{2} m v^{2}$ and $\kappa_{w}:=\frac{1}{2} I w^{2}$,

$$
\begin{aligned}
\frac{d}{d t} \kappa_{v} & =u_{v} v+m d \dot{\theta} w v \\
\frac{d}{d t} \kappa_{w} & =u_{w} w-m d \dot{\theta} v w
\end{aligned}
$$

where the last terms in (4) and (5), which represent the coupling effects, are summed to be zero (i.e. define energetically conservative internal energy shuffling). These coupling terms disappear if $d=0$ (i.e. $\left(x_{c}, y_{c}\right)$ and $\left(x_{o}, y_{o}\right)$ coincide with each other) or if the Coriolis terms $Q \nu$ in (2) are canceled out as done in the next Sec. III. Then, we will get (4)-(5) without the last terms. This implies that the (decoupled) dynamics of $v$ and $w$ will then individually possess the passivity property similar to (3).

\section{Passivity-Based Switching Control Design for POSTURE STABILIZATION}

In this section, we design the switching control $u$ in (2) s.t. $\lim _{t \rightarrow \infty}(x(t), y(t))$ and the desired position $\left(x_{d}, y_{d}\right)$ is close enough in the sense that a certain distance measure between these two point is less than or equal to a user-specific performance specification $\delta_{o}>0$ and $\theta(t) \rightarrow \theta_{d}$. Without losing generality, here, we assume that $\left(x_{d}, y_{d}, \theta_{d}\right)=(0,0,0)$.

Now, we design the control law $\left(u_{v}, u_{w}\right)$ s.t.

$\left(\begin{array}{l}u_{v} \\ u_{w}\end{array}\right)=\left[\begin{array}{cc}0 & -m d \dot{\theta} \\ m d \dot{\theta} & 0\end{array}\right]\left(\begin{array}{c}v \\ w\end{array}\right)-\left(\begin{array}{c}b v+\frac{\partial \varphi_{v}}{\partial x} \mathrm{c} \theta+\frac{\partial \varphi_{v}}{\partial y} \mathrm{~s} \theta \\ b_{w} \dot{\theta}+k_{w}\left(\theta-\theta_{\sigma(t)}\right)\end{array}\right)$

where $\mathrm{s} \theta=\sin \theta, \mathrm{c} \theta=\cos \theta, b, b_{w}>0$ are the damping gains, $k_{w}>0$ is the spring gain, and $\sigma(t) \in\{1,2\}$ is the switching signal s.t. $\theta_{1}=0$ and $\theta_{2}=\theta_{o}$ with $\theta_{o} \neq \theta_{1}+n \pi$ being a constant $(n=0, \pm 1, \pm 2, \ldots)$. Switching law for $\sigma(t)$ will be designed below (see Theorem 1). Here, $\varphi_{v}(x, y) \geq 0$ is a smooth navigation potential function [14] defined on the $(x, y)$-plane s.t. 1) $\varphi_{v}(x, y)=0$ if and only if $\left.(x, y)=0 ; 2\right)$ $\left(\frac{\partial \varphi_{v}}{\partial x}, \frac{\partial \varphi_{v}}{\partial y}\right)=0$ if and only if $(x, y)=0$; and 3$)$ for any finite constants $l \geq 0$, the level set

$$
\mathcal{L}_{l}:=\left\{(x, y) \in \Re^{2} \mid \varphi_{v}(x, y) \leq l\right\}
$$

is a compact set containing $(0,0)$ and $\mathcal{L}_{l_{1}} \subseteq \mathcal{L}_{l_{2}}$ if $l_{2} \geq$ $l_{1} \geq 0$. In addition to the stabilization $(x, y) \rightarrow(0,0)$, this navigation potential $\varphi_{v}(x, y)$ can also incorporate other control objectives such as obstacle avoidance [23], although how to design such a potential field without unwanted local minima is beyond the scope of this paper. Here, we want to emphasize that this potential function $\varphi_{v}(x, y)$ can be designed without considering the nonholonomic constraints as if the given system is just a (unconstrained) point mass on $(x, y)$ plane. Thus, we can use many of already available results for the generation of this $\varphi_{v}(x, y)$ (e.g. [14]).
Under this control (6), the closed-loop dynamics of (2) becomes

$$
\begin{array}{r}
m \dot{v}+b v+\frac{\partial \varphi_{v}}{\partial x} \mathrm{c} \theta+\frac{\partial \varphi_{v}}{\partial y} \mathrm{~s} \theta=0 \\
I \ddot{\theta}+b_{w} \dot{\theta}+k_{w}\left(\theta-\theta_{\sigma(t)}\right)=0
\end{array}
$$

where, due to the decoupling control in (6) (first term) and the fact that $\varphi_{v}$ is a function of $(x, y)$, these two dynamics (8)-(9) are energetically decoupled from each other: 1) for the $v$-dynamics (8), the total energy $V_{v}:=\kappa_{v}+\varphi_{v}(x, y)$ is a function of only $(v, x, y)$ and, from (4),

$$
\frac{d}{d t} V_{v}=-b v^{2}-\frac{\partial \varphi_{v}}{\partial x} v \mathrm{c} \theta-\frac{\partial \varphi_{v}}{\partial y} v \mathrm{~s} \theta+\frac{d \varphi_{v}}{d t}=-b v^{2}
$$

with $v \mathrm{c} \theta=\dot{x}$ and $v \mathrm{~s} \theta=\dot{y}$ (from (1)); and 2) for the $w$ dynamics (9), the total energy $V_{w}:=\kappa_{w}+\varphi_{w}$ with $\varphi_{w}=$ $k_{w}\left(\theta-\theta_{\sigma(t)}\right)^{2} / 2$ is a function of only $\left(\theta, \theta_{\sigma(t)}, w\right)$ and, from (5), $d V_{w} / d t=-b_{w} w^{2}$ between two consecutive switchings. Due to this energetic decoupling between (8)-(9), switchings in $\theta_{\sigma(t)}$, which induce jumps in $V_{w}$, neither change the value of $V_{v}$ at the switching nor affect the dissipation relation of (10). Also, note that, by the decoupling control in (6), the $w$ dynamics (9) is completely decoupled from the $v$-dynamics (8).

Proposition 1 Suppose that $\sigma(t)$ is fixed with $\sigma(t)=\sigma \forall t \geq$ 0 . Then, under the control (6), $\left(v, w, \theta-\theta_{\sigma}\right) \rightarrow 0$ and the the robot's $(x, y)$-position converges to the following submanifold

$$
\mathcal{M}_{\sigma}:=\left\{(x, y) \in \Re^{2} \mid \frac{\partial \varphi_{v}}{\partial x} \mathrm{c} \theta_{\sigma}+\frac{\partial \varphi_{v}}{\partial y} \mathrm{~s} \theta_{\sigma}=0\right\} .
$$

Proof: From (10), we have $V_{v}(t) \leq V_{v}(0)$. Therefore, for all $t \geq 0, v$ (also, $\dot{x}, \dot{y}$ from (1)) is bounded and $(x, y) \in \mathcal{L}_{V_{v}(0)}$, where $\mathcal{L}_{V_{v}(0)}$ is a compact set containing $(0,0)$. Also, for (9), if $\sigma(t)=\sigma \forall t \geq 0$, we have $d V_{w} / d t=-b_{w} w^{2} \forall t \geq 0$, thus, $\left(w, \theta-\theta_{\sigma}\right)$ is bounded $\forall t \geq 0$, too. Therefore, applying LaSalle's Theorem [24] to (8)-(9) with $V:=V_{v}+V_{w}$ and $d V / d t=-b v^{2}-b_{w} w^{2}$ for all $t \geq 0$, we have $\left(v, w, \theta-\theta_{\sigma}\right) \rightarrow 0$ and $\frac{\partial \varphi_{v}}{\partial x} \mathrm{c} \theta_{\sigma}+\frac{\partial \varphi_{v}}{\partial y} \mathrm{~s} \theta_{\sigma} \rightarrow 0$.

The main idea of our switching control is to make the system to move back and forth between these two submanifolds $\mathcal{M}_{1}$ and $\mathcal{M}_{2}$ (by switching $\theta_{\sigma}$ between $\theta_{1}$ and $\theta_{2}$ ), while guaranteeing that the navigation potential $\varphi_{v}$ is strictly decreasing during these inter-switching moves. Here, note that $\mathcal{M}_{1}$ and $\mathcal{M}_{2}$ intersect with each other only at $(x, y)=0$.

Now, suppose that two consecutive switchings occur at $t_{i}$ and $t_{i+1}$ and $\sigma(t)=\sigma_{i}$ for $I_{i}:=\left[t_{i}, t_{i+1}\right)$. Then, for this time-interval $I_{i}$, by integrating (10), we have

$$
\begin{aligned}
\varphi_{v}\left(t_{i}\right)-\varphi_{v}\left(t_{i+1}\right) & =\kappa_{v}\left(t_{i+1}\right)-\kappa_{v}\left(t_{i}\right)+\int_{t_{i}}^{t_{i+1}} b v^{2} d t \\
& \geq-\kappa_{v}\left(t_{i}\right)+\int_{t_{i}}^{t_{i+1}} b v^{2} d t
\end{aligned}
$$

since $\kappa_{v}(t) \geq 0$. Thus, if we can ensure that, during the system's inter-switching move between the two submanifolds, the 
energy dissipation via the damping $b$ is strictly larger than the initial kinetic energy $\kappa_{v}\left(t_{i}\right), \varphi_{v}(t)$ will be strictly decreasing between the switchings, thus, we can achieve $\varphi_{v}(t) \rightarrow 0$. This would be trivially achieved if $\kappa_{v}\left(t_{i}\right)=0$. However, detecting this requires perfect velocity sensing and, even with that, it requires the time-interval between two consecutive switchings be infinite.

The next Lemma shows that, if the wheeled mobile robot's moving distance is large enough and the initial velocity is small enough, the damping can always dissipate all the initial kinetic energy.

Lemma 1 Suppose that, on the (x,y)-plane, the wheeled mobile robot under the control (6) moves from $\left(x_{0}, y_{0}\right)$ to $\left(x_{1}, y_{1}\right)$ with the distance between them being $D>0$. Suppose further that the robot's $(x, y)$-trajectory is twice-differentiable. Then, if $\kappa_{v}\left(t_{0}\right)<2 b^{2} D^{2} / m$,

$$
\int_{t_{0}}^{t_{1}} b v^{2} d t>\kappa_{v}\left(t_{0}\right)
$$

where $t_{0}$ and $t_{1}$ are the initial and (unspecified) final times.

Proof: This is trivially ensured if $\kappa_{v}\left(t_{0}\right)=0$, since $D>0$. Now, suppose that $\kappa_{v}\left(t_{0}\right)>0$. Then, from the standard result of the calculus of variation [25], among the twice-differentiable trajectories $a(t)=\left(x_{a}(t), y_{a}(t)\right)$ on $\Re^{2}$ connecting $\left(x_{0}, y_{0}\right)$ and $\left(x_{1}, y_{1}\right)$, the one that extremizes $\int b v_{a}^{2} d t=\int b\left(\dot{x}_{a}^{2}+\dot{y}_{a}^{2}\right) d t$ is given by the Lagrangian equation, that is, in this case, simply given by $\ddot{x}_{a}=\ddot{y}_{a}=0$. This implies that this extremizing trajectory (i.e. geodesics) is the straight line connecting the two points with constant velocity (and kinetic energy) along the line. This extremizer is also the minimizer, since we can find another curves with higher damping dissipation (e.g. fast oscillatory curve).

Thus, if the wheeled mobile robot moves from $\left(x_{0}, y_{0}\right)$ to $\left(x_{1}, y_{1}\right)$ along a twice-differentiable trajectory with $\kappa_{v}\left(t_{0}\right)>$ 0 , we have: with $\bar{v}_{a}:=\sqrt{2 \kappa_{v}\left(t_{0}\right) / m}$,

$$
\int_{t_{0}}^{t_{1}} b v^{2} d t \geq \min _{a(t)} \int b v_{a}^{2} d t=b \bar{v}_{a}^{2} \frac{D}{\bar{v}_{a}}=b D \sqrt{\frac{2 \kappa_{v}\left(t_{0}\right)}{m}}
$$

which is strictly larger than $\kappa_{v}\left(t_{0}\right)$ if $0<\kappa_{v}\left(t_{0}\right)<2 b^{2} D^{2} / m$.

This Lemma 1, thus, enables us to switch even when $\kappa_{v} \neq 0$ while enforcing strict decrease of $\varphi_{v}$ between the switchings, providing that the switching occurs when the velocity is small and the moving distance of the robot between two consecutive switchings is not small. Here, the former condition can be ensured simply by waiting for the system to slow down enough into the switching submanifold (see Proposition 1), while the latter by designing the two submanifolds $\mathcal{M}_{1}$ and $\mathcal{M}_{2}$ far enough from each other. In many cases, this separation would be possible (at least locally) except very near the origin, where $\mathcal{M}_{1}$ and $\mathcal{M}_{2}$ intersect with each other.

The Lemma 1 is directly applicable for the wheeled mobile robot between two consecutive switchings, since, with $\theta_{\sigma(t)}$

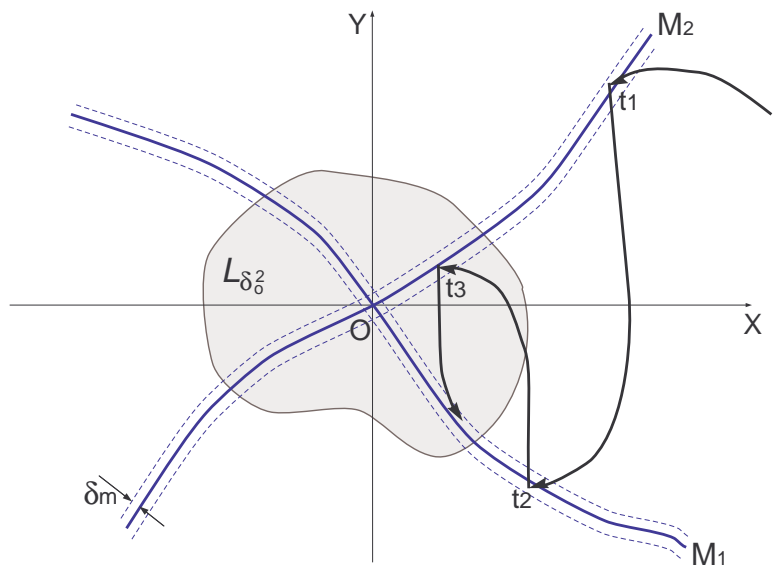

Fig. 2. Illustration of level set $\mathcal{L}_{\delta_{o}^{2}}$, submanifolds $\mathcal{M}_{1}$ and $\mathcal{M}_{2}$, and their strips with thickness of $\delta_{m}>0$ on the $(x, y)$-plane. Also, shown is an exemplary system trajectory with switching times.

being a constant, both the dynamics (2) and the control (6) are smooth, therefore, the system's $(x, y)$-trajectory is also smooth. This is all we need for our switching control design. However, it is also interesting to see that this Lemma 1 is still valid even in the presence of switchings. To check this, recall from (1) that $\dot{x}=v \mathrm{c} \theta$. Thus, $\ddot{x}=\dot{v} \mathrm{c} \theta-v \mathrm{~s} \theta \dot{\theta}$, where, from (8)-(9), $\dot{v}, \dot{\theta}$ are both bounded and continuous. Therefore, $\ddot{x}(t)$ is also bounded and continuous $\forall t \geq 0$. Similar fact can be shown for $\ddot{y}(t)$, too. This implies that, although the non-smooth switching $\theta_{\sigma(t)}$ occurs in (9), the robot's $(x, y)$ trajectory is always twice-differentiable, therefore, Lemma 1, which only requires that candidate trajectory $a(t)$ is twicedifferentiable, still holds regardless of the switching.

We now present our main result with passivity (or energy) based switching logic.

Theorem 1 Suppose that the navigation potential $\varphi_{v}$ is designed to satisfy the following condition: $\exists c_{m}>0$ s.t., for any $(x, y) \notin \mathcal{L}_{\delta_{o}^{2}}$, if $(x, y) \in \overline{\mathcal{M}}_{i}$,

$$
\operatorname{dist}\left((x, y), \overline{\mathcal{M}}_{j}\right) \geq c_{m} \sqrt{\varphi_{v}(x, y)}
$$

where $\mathcal{L}_{\delta_{o}^{2}}$ is the level set of $\varphi_{v}$ in (7) with $\delta_{o}>0$ being a user-specified performance measure, $\overline{\mathcal{M}}_{k}$ is the "strip" of $\mathcal{M}_{k}$ with a thickness $\delta_{m}>0$ s.t. $c_{m} \delta_{o} \gg \delta_{m}(k=1,2$, see Fig. 2), and $(i, j) \in\{(1,2),(2,1)\}$. Trigger the switching at a time $t>0$, if

1) $\kappa_{v}(t) \leq 2 b^{2} c_{m}^{2} \delta_{o}^{2} / m$; and

2) $\operatorname{dist}\left((x(t), y(t)), \mathcal{M}_{\sigma\left(t^{-}\right)}\right) \leq \delta_{m}$; and

3) a) $(x(t), y(t)) \notin \mathcal{L}_{\delta_{o}^{2}}$; or

b) $(x(t), y(t)) \in \mathcal{L}_{\delta_{o}^{2}}$ and $\sigma\left(t^{-}\right) \notin 1$.

Then, $\lim _{t \rightarrow \infty}(x(t), y(t)) \in \mathcal{L}_{\delta_{o}^{2}}$ and $\theta(t) \rightarrow \theta_{d}$. Also, once $(x(t), y(t)) \in \mathcal{L}_{\delta_{o}^{2}}$, at most one more switching occurs.

Proof: The system's initial condition is given by $(\dot{q}(0), q(0), \sigma(0))$, where $\sigma(0) \in\{1,2\}$. Then, from Prop. $1, \theta \rightarrow \theta_{\sigma(0)}$ and $(x, y) \rightarrow \mathcal{M}_{\sigma(0)}$. Let us denote the time 
when the switching conditions 1)-2) are satisfied by $t_{1}>0$, i.e. $\kappa_{v}\left(t_{1}\right) \leq 2 b^{2} c_{m}^{2} \delta_{o}^{2} / m$ and $\operatorname{dist}\left(\left(x\left(t_{1}\right), y\left(t_{1}\right)\right), \overline{\mathcal{M}}_{\sigma(0)}\right) \leq$ $\delta_{m}$. Consider the following two cases separately, 1) when $\left(x\left(t_{1}\right), y\left(t_{1}\right)\right) \notin \mathcal{L}_{\delta_{o}^{2}} ;$ and 2$)$ otherwise.

1) Outside of $\mathcal{L}_{\delta_{o}^{2}}$ : If $\left(x\left(t_{1}\right), y\left(t_{1}\right)\right) \notin \mathcal{L}_{\delta_{o}^{2}}$, according to the switching logic, there will be a switching at this $t_{1}$ s.t. $\sigma\left(t_{1}\right)=$ 1 if $\sigma(0)=2$ or $\sigma\left(t_{1}\right)=2$ if $\sigma(0)=1$. Then, following Prop. $1,(x, y) \rightarrow \overline{\mathcal{M}}_{\sigma\left(t_{1}\right)}$ and $\theta \rightarrow \theta_{\sigma\left(t_{1}\right)}$. Denote the time when the system again satisfies the above switching conditions 1)-2) by $t_{2}>t_{1}$, i.e. the system converges into $\overline{\mathcal{M}}_{\sigma\left(t_{1}\right)}$ and slows down enough. During this time-interval $I_{1}:=\left[t_{1}, t_{2}\right)$, the potential function $\varphi_{v}(t)$ is strictly decreasing, since, following (11), we have

$$
\varphi_{v}\left(t_{1}\right)-\varphi_{v}\left(t_{2}\right) \geq-\kappa_{v}\left(t_{1}\right)+\int_{t_{1}}^{t_{2}} b v^{2} d t>0
$$

where the last inequality is a direct consequence of Lemma 1 , since, from (13) with $\left(x\left(t_{1}\right), y\left(t_{1}\right)\right) \notin \mathcal{L}_{\delta^{2}}$, the moving distance $D_{I_{1}}>0$ of the wheeled mobile robot from $\left(x\left(t_{1}\right), y\left(t_{1}\right)\right) \in \overline{\mathcal{M}}_{\sigma(0)}$ to $\left(x\left(t_{2}\right), y\left(t_{2}\right)\right) \in \overline{\mathcal{M}}_{\sigma\left(t_{1}\right)}$ satisfies $D_{I_{1}} \geq c_{m} \sqrt{\varphi_{v}\left(x\left(t_{1}\right), y\left(t_{1}\right)\right)}>c_{m} \delta_{o}$, but, $\kappa_{v}\left(t_{1}\right) \leq$ $2 b^{2} c_{m}^{2} \delta_{o}^{2} / m<2 b^{2} D_{I_{1}}^{2} / m$. If $\left(x\left(t_{2}\right), y\left(t_{2}\right)\right) \notin \mathcal{L}_{\delta_{o}^{2}}$, another switching will be triggered.

By continuing this process, since the navigation potential $\varphi_{v}\left(t_{i}\right)$ is strictly decreasing, a sequence of the times can be generated $\left(t_{1}, t_{2}, \ldots, t_{n-1}, t_{n}\right)$, where $\left(x\left(t_{k}\right), y\left(t_{k}\right)\right) \notin \mathcal{L}_{\delta_{o}^{2}} \forall k=1,2, \ldots, n-1$ (i.e. $t_{1}, t_{2}, \ldots, t_{n-1}$ are the switching times) and, at time $t_{n}$ (switching time candidate: $n \geq 2$ ), the switching conditions 1)-2) are satisfied with $\left(x\left(t_{n}\right), y\left(t_{n}\right)\right) \in \mathcal{L}_{\delta_{o}^{2}}$. Then, it becomes the case 2) discussed below.

2) Inside of $\mathcal{L}_{\delta_{0}^{2}}$ : Now, suppose that, at some $t_{n}$ with $n \geq 1,\left(x\left(t_{n}\right), y\left(t_{n}\right)\right) \in \mathcal{L}_{\delta_{o}^{2}}$ and the two conditions 1)-2) are satisfied. Then, if $\sigma\left(t_{n}^{-}\right)=1$, we are done and no more switching will be triggered. If $\sigma\left(t^{-}\right)=2$, to correct the orientation, another switching will be activated at $t_{n}$ with $\sigma\left(t_{n}\right)=1$ and the system will again converge into $\overline{\mathcal{M}}_{1}$. Denote by $t_{n+1}$ the time when the system again satisfies the two switching conditions 1)-2) in $\overline{\mathcal{M}}_{1}$. In this case, $\left(x\left(t_{n+1}\right), y\left(t_{n+1}\right)\right)$ must be still contained in $\mathcal{L}_{\delta_{o}^{2}}$. This can be shown by the following contradiction. Suppose that $\left(x\left(t_{n+1}\right), y\left(t_{n+1}\right)\right) \notin \mathcal{L}_{\delta_{o}^{2}}$. Then, from (13), the wheeled mobile robot should move the distance strictly larger than $c_{m} \delta_{o}, \quad$ since $\operatorname{dist}\left(\left(x\left(t_{n}\right), y\left(t_{n}\right)\right),\left(x\left(t_{n+1}\right), y\left(t_{n+1}\right)\right)\right) \geq$ $c_{m} \sqrt{\varphi_{v}\left(x\left(t_{n+1}\right), y\left(t_{n+1}\right)\right)}>c_{m} \delta_{o}$. Thus, with $\kappa_{v}\left(t_{n}\right) \leq 2 b^{2} c_{m}^{2} \delta_{o}^{2} / m$, the inequality (14) still holds and $\varphi_{v}\left(t_{n+1}\right)<\varphi_{v}\left(t_{n}\right)$. This implies that $\left(x\left(t_{n+1}\right), y\left(t_{n+1}\right)\right) \in \mathcal{L}_{\varphi_{v}\left(t_{n+1}\right)} \subset \mathcal{L}_{\varphi_{v}\left(t_{n}\right)} \subset \mathcal{L}_{\delta_{o}^{2}}$, which is contradictory to the above supposition. Therefore, we have $\lim _{t \rightarrow \infty}(x(t), y(t)) \in \mathcal{L}_{\delta_{o}^{2}}$ and $\theta(t) \rightarrow \theta_{d}$. This completes the proof.

If the objective is only posture stabilization (without obstacle avoidance), the frequently-used quadratic function $k_{v}\left(x^{2}+\right.$ $\left.y^{2}\right)$ with $k_{v}>0$ can be directly used as the navigation

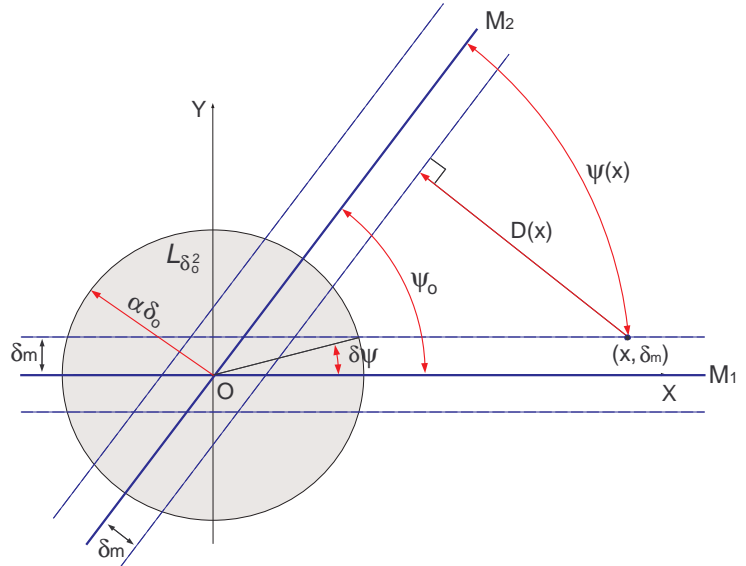

Fig. 3. Illustration of some geometric entities of $\varphi_{v}(x, y)=k_{v}\left(x^{2}+\right.$ $\left.y^{2}\right) / 2$ : with $\left.\alpha=\sqrt{2 / k_{v}}, 1\right) \mathcal{L}_{\delta_{o}^{2}}$ is circle with radius $\alpha \delta_{o}>0$;2) if $(x, y) \in \mathcal{M}_{1}-\mathcal{L}_{\delta_{o}^{2}},|y| \leq \delta_{m}$ and $|x|>\alpha \delta_{o}$; and 3) $\sin \psi(x)=$ $\left(D(x)+\delta_{m}\right) / \sqrt{x^{2}+\delta_{m}^{2}}$ and $0<\psi_{o}-\delta \psi \leq \psi(x) \leq \psi_{o} \leq \pi / 2$. Here, we conveniently choose an arbitrarily $x$-axis as aligned to $\mathcal{M}_{1}$.

potential $\varphi_{v}$. This is because 1) this quadratic function satisfies all the conditions for $\varphi_{v}$ given in the beginning of this Sec. III; and 2) if we set $\alpha \delta_{o} \gg \delta_{m}$ and choose $\theta_{1}, \theta_{2}$ s.t. the two switching submanifolds $\mathcal{M}_{i}=\left\{(x, y) \mid k_{v} x \mathrm{c} \theta_{i}+\right.$ $\left.k_{v} y \mathrm{~s} \theta_{i}=0\right\}$ (i.e. straight line obtained by rotating $y$-axis by $\theta_{i}$ counterclockwise w.r.t. origin) are far enough from each other, it also ensures the condition (13) with $c_{m} \leq$ $\alpha\left[\sin \left(\psi_{o}-\delta \psi\right)-\sin \delta \psi\right]$, where $\alpha=\sqrt{2 / k_{v}}, 0<\psi_{o} \leq \pi / 2$ is the angle between $\mathcal{M}_{1}, \mathcal{M}_{2}$, and $\delta \psi=\sin ^{-1}\left(\delta_{m} /\left(\alpha \delta_{o}\right)\right)$. This is because, from Fig. 3, if $(x, y) \in \overline{\mathcal{M}}_{1}-\mathcal{L}_{\delta_{o}^{2}}$,

$$
\begin{aligned}
\frac{\operatorname{dist}\left((x, y), \overline{\mathcal{M}}_{2}\right)}{\sqrt{\varphi_{v}(x, y)}} & \geq \alpha \frac{D(x)}{\sqrt{x^{2}+\delta_{m}^{2}}} \\
& =\alpha\left(\sin \psi(x)-\frac{\delta_{m}}{\sqrt{\alpha^{2} \delta_{o}^{2}+\delta_{m}^{2}}}\right) \\
& >\alpha\left[\sin \left(\psi_{o}-\delta \psi\right)-\sin \delta \psi\right] .
\end{aligned}
$$

On the other hand, in practice, the switching conditions 1)2) in Theorem 1 can be easily ensured by separating two consecutive switchings by a large enough dwell-time $\tau_{D}>0$ [26].

The decoupling control in (6) is necessary here, since, if we omit it, energy can be transferred between (8) and (9) via the coupling terms in (4)-(5). Then, some portion of the $\theta$-spring energy $\varphi_{w}=\frac{1}{2} k_{w}\left(\theta-\theta_{\sigma(t)}\right)^{2}$, which jumps at every switching, may flow back to the navigation potential $\varphi_{v}$ and recharge it. If this amount is more than the damping dissipation via $b$, we may lose the strict decrease of $\varphi_{v}(t)$ between switchings. More detailed analysis on the effect of this (uncompensated or partially-compensated) coupling is a topic for future research.

\section{SIMULATION}

For this simulation, we use the quadratic navigation potential $\varphi_{v}(x, y)=\frac{1}{2} k_{v}\left(x^{2}+y^{2}\right)$ with $k_{v}>0$. We also use a long 
enough dwell-time $\tau_{D}>0$ to ensure the switching conditions 1)-2) of Theorem 1. For the first simulation, we choose $\theta_{1}=0$ and $\theta_{2}=\pi / 2$ so that the two submanifolds $\mathcal{M}_{1}$ and $\mathcal{M}_{2}$ are respectively given by the $y$-axis and $x$-axis. As stated in the paragraph after Theorem 1 , these chosen $\varphi_{v}$ and $\theta_{1}, \theta_{2}$ are legitimate for use in our switching control. Simulation results are presented in Fig. 4. Total thirty-six switchings are occurred. After the thirty-fifth switching (around 17.5sec), the system's $(x, y)$-trajectory enters into the desired level set, but, its orientation is not correct. So, another thirty-sixth switching occurs (around 18sec.) to correct the orientation. After this last switching, the system's $(x, y)$-position is in the desired level set, the orientation converges to the target value, and no more switching occurs.

For the second simulation, we use $\theta_{1}=-\pi / 2$ and $\theta_{2}=$ $\pi / 2$, thus, $\mathcal{M}_{1}$ and $\mathcal{M}_{2}$ are the same: both of them are given by $k_{v} y=0$ (i.e. $x$-axis). However, by tuning the gains, we make the $v$-dynamics (8) much faster than that of $w$ (9), so that the robot's $(x, y)$-trajectory converges on the straight line $\mathcal{M}_{\theta(t)}=\left\{(x, y) \mid k_{v} x \mathrm{c} \theta(t)+k_{v} x \mathrm{~s} \theta(t)=\right.$ $0\}$ fast enough, as this line rotates slowly back and force between the positive $x$-axis and the negative $x$-axis. By doing so, although $\mathcal{M}_{1}=\mathcal{M}_{2}$, we can ensure that, between the switchings, the robot moves between the two strips on the positive $x$-axis and the negative $x$-axis separated by $\mathcal{L}_{\delta_{0}^{2}}$. This ensures the condition (13), since, if $(x, y) \in \mathcal{M}_{i}-$ $\mathcal{L}_{\delta_{o}^{2}}$ and $\sqrt{2 / k_{v}} \delta_{o} \gg \delta_{m}, \operatorname{dist}\left((x, y), \overline{\mathcal{M}}_{j}\right) / \sqrt{\varphi_{v}(x, y)} \geq$ $\sqrt{2 / k_{v}}\left(|x|+\delta_{m}\right) / \sqrt{x^{2}+\delta_{m}^{2}}>\sqrt{2 / k_{v}}$, thus, any $0<c_{m} \leq$ $\sqrt{2 / k_{v}}$ would work to enforce (13). Results for this simulation are shown in Fig. 5. Compared to the results in Fig. 4, much less number of switchings (only ten switchings) is required to move into the desired level set, since the moving distance between two switchings is larger than that of Fig. 4.

Similar to the second simulation, we may slowly rotate the submanifold more than $2 \pi$. For this, again, by tuning the dynamics of $v$ much faster than that of $w$, we could get an even faster convergence and less number of switchings. A detailed analysis and exposition for this rotating submanifold result will be reported in a future publication.

\section{Application to Multiple Wheeled Mobile ROBOTS COORDINATION}

In this section, as one example of extensions to more general problems/systems, we apply the passivity-based switching control for the coordination problem of multiple wheeled mobile robots. No motion planning is necessarily here. More detailed exposition will be reported in future publications.

Consider $n$ (strongly-connected) wheeled mobile robots, and define $p_{e}:=\left(x_{e}, y_{e}\right) \in \Re^{2(n-1)}$ and $\theta_{e} \in \Re^{n-1}$, with $\star_{e}:=\left(\star_{1}-\star_{2}, \ldots, \star_{n-1}-\star_{n}\right) \in \Re^{n-1}$. Then, for simplicity (while without losing generality), by the coordination, we mean $\left(p_{e}, \theta_{e}\right) \rightarrow 0$. Then, for each $k$-th agent, we design its control to be (6) with its second term replaced by

$$
-\left(\begin{array}{c}
b v_{k}+\frac{\partial \bar{\varphi}_{v}}{\partial x_{k}} \mathrm{c} \theta_{k}+\frac{\partial \bar{\varphi}_{v}}{\partial y_{k}} \mathrm{~s} \theta_{k} \\
b_{w} \dot{\theta}_{k}+\frac{\partial \bar{\varphi}_{w}}{\partial \theta_{k}}+k_{w}\left(\theta_{k}-\theta_{\sigma(t)}\right)
\end{array}\right)
$$

where $\bar{\varphi}_{v}\left(p_{e}\right)$ and $\bar{\varphi}_{w}\left(\theta_{e}\right)$ are (smooth) navigation potentials defined on the $p_{e}$ and $\theta_{e}$ spaces respectively.

Then, similar to Prop. 1, using Barbalat's lemma with smoothness of suitable terms, we can show that, with fixed $\sigma(t)=\sigma$, the system converges in the $p_{e}$-space to the submanifold $\mathcal{T}_{\sigma}:=\left\{p_{e} \mid \frac{\partial \bar{\varphi}_{v}}{\partial x} \mathrm{c} \theta_{\sigma}+\frac{\partial \bar{\varphi}_{v}}{\partial y} \mathrm{~s} \theta_{\sigma}=0\right\}$, where $\frac{\partial \bar{\varphi}_{v}}{\partial \star}:=\left(\frac{\partial \bar{\varphi}_{v}}{\partial \star_{1}}, \ldots, \frac{\partial \bar{\varphi}_{v}}{\partial \star_{n}}\right) \in \Re^{n}$. Also, being unconstrained, $\theta_{k} \rightarrow \theta_{\sigma}$. Moreover, between switchings, similar to (11), we have

$$
\begin{aligned}
\bar{\varphi}_{v}\left(t_{i}\right)-\bar{\varphi}_{v}\left(t_{i+1}\right) & \geq-\bar{\kappa}_{v}\left(t_{i}\right)+\sum_{k=1}^{n} \int_{t_{i}}^{t_{i+1}} b v_{k}^{2} d t \\
& \geq-\bar{\kappa}_{v}\left(t_{i}\right)+\int_{t_{i}}^{t_{i+1}} \bar{b}\left\|v_{e}\right\|^{2} d t
\end{aligned}
$$

where $\bar{b}>0, v_{e}=d p_{e} / d t$ (i.e. system velocity on $p_{e}$-space), and $\bar{\kappa}_{v}=\sum \frac{1}{2} m_{k} v_{k}^{2}$. Here, we can obtain the last inequality by using the passive decomposition [13].

Therefore, we can achieve similar results as those in Sec. III on the $p_{e}$-space. In other words, if we design $\bar{\varphi}_{v}$ on the $p_{e}$-space s.t. the switching submanifolds $\mathcal{T}_{i}$ are far enough from each other and trigger the switchings when $\bar{\kappa}_{v}$ is small enough, we can ensure that $\theta_{e} \rightarrow 0$ and $p_{e}$ approaches to 0 within some user-specific performance bound. See Fig. 6 for simulation results of this as applied to four wheeled mobile robots. Here, our coordination control is centralized, although it can be partially decentralized by defining $\bar{\varphi}_{v}$ as the sum of the potential between two robots. Its complete decentralization is beyond the scope of this paper and will be published elsewhere.

\section{Summary AND Future WORKS}

In this paper, we propose a novel passivity-based switching control law for the posture stabilization of a wheeled mobile robot. The proposed control law is derived using the fundamental (open-loop) passivity property, which has been extensively used in other control problems in robotics, but not been so at all for systems with nonholonomic constraints.

Since it is based on fairly intrinsic concepts and entities (e.g. passivity, dissipation, decoupling, submanifolds), we believe that our proposed framework could be extended for more general control problems (e.g. coordination problem of multiple wheeled mobile robots as presented in Sec. V), or even further, control of general mechanical systems with nonholonomic constraints on a differential manifold. The latter may require that those systems have dynamics/energetic structure similar to, but probably more generalized than, that of the wheeled mobile robot. Real implementation of this passivity-based switching control and its experimental comparison with other schemes may also further shed lights on its strength/weakness and robustness/practicality as well.

We also wish that this work serves as an initiating step toward fully utilizing the passivity property in the control of wheeled mobile robots and more general robotic systems with nonholonomic constraints. 


\section{REFERENCES}

[1] M. Peshkin and J. E. Colgate. Cobots. Industrial Robot, 26(5):335-341, 1999.

[2] C.R. Weisbin and D. Lavery. Nasa rover and telerobotics technology program. IEEE Robotics \& Automation Magazine, pages 14-21, December 1994.

[3] S. P. Levine, D. A. Bell, L. A. Jaros, R. C. Simpson, Y. Koren, and J. Borenstein. The navchair assistive wheelchair navigation system. IEEE Transactions on Rehabilitation Engineering, 7(4):443-451, 1999.

[4] J. Cortes, S. Martinez, T. Karatas, and F. Bullo. Coverage control for mobile sensing networks. IEEE Transactions on Robotics and Automation, 20(2):243-255, 2004.

[5] R. M. Murray, Z. Li, and S. S. Sastry. A mathematical introduction to robotic manipulation. CRC, Boca Ranton, FL, 1993.

[6] R. W. Brockett. Asymptotic stability and feedback stabilization. In R. W. Brockett, R. S. Milman, and H. J. Sussmann, editors, Differential Geometric Control Theory, pages 181-191, Boston, MA, 1983. Birkhauser.

[7] J. C. Willems. Dissipative dynamical systems part1: general theory. Arch. Rational Mech. Anal., 45(22):321-351, 1972.

[8] M. W. Spong, S. Hutchinson, and M. Vidyasaga. Robot modeling and control. John Wiley \& Sons, Hoboken, NJ, 2006.

[9] J. E. Colgate and G. Schenkel. Passivity of a class of sampled-data systems: application to haptic interfaces. Journal of Robotic Systems, 14(1):37-47, 1997.

[10] D. J. Lee and M. W. Spong. Passive bilateral teleoperation with constant time delay. IEEE Transactions on Robotics, 22(2):269-281, 2006.

[11] M. W. Spong, J. K. Holm, and D. J. Lee. Passivity-based control of biped locomotion. IEEE Robotics \& Automation Magazine. To appear.

[12] M. Arcak. Passivity as a design tool for group coordination. In Proceeding of the American Control Conference, pages 29-23, 2006.

[13] D. J. Lee and P. Y. Li. Passive decomposition approach to formation and maneuver control of multiple agents with inertias. ASME Journal of Dynamic Systems, Measurement \& Control. To appear.

[14] E. Rimon and D. E. Koditschek. Exact robot navigation using artificial potential functions. IEEE Transactions on Robotics and Automation, 8(5):501-518, 1992

[15] C. Canudas de Wit and O. J. Sordalen. Exponential stabilization of mobile robots with nonholonomic constraints. IEEE Transactions on Automatic Control, 13(11):1791-1797, 1992.

[16] C. Samson. Time-varying feedback stabilization of car-like wheeled mobile robots. International Journal of Robotics Research, 12(1):5564, 1993.

[17] R. Fierro and F. L. Lewis. Control of a nonholonomic mobile robot: backstepping kinematics into dynamics. Journal of Robotic Systems, 14(3):149-163, 1997.

[18] I. Kolmanovsky, M. Reyhanoglu, and N. H. McClamroch. Switched mode feedback control laws for nonholonomic systems in extended power form. Systems \& Control Letters, 27:29-36, 1996.

[19] J. P. Hespanha and A. S. Morse. Stabilization of nonholonomic integrators via logic-based switching. Automatica, 35:385-393, 1999.

[20] J. P. Hespanha, D. Liberzon, and A. S. Morse. Logic-based switching control of a nonholonomic system with parametric modeling uncertainty. Systems \& Control Letters, 38:167-177, 1999.

[21] A. Astolfi. Exponential stabilization of a wheeled mobile robots via discontinuous control. ASME Journal of Dynamic Systems, Measurement \& Control, 121(1):121-126, 1999.

[22] K. Fujimoto and T. Sugie. Stabilization of hamiltonian systems with nonholonomic constraints based on time-varying generalized canonical transformation. Systems \& Control Letters, 44:309-319, 2001.

[23] H. G. Tanner, A. Jadbabaic, and G. J. Pappas. Flocking in teams of nonholonomic agents. In S. Morse, N. Leonard, and V. Kumar, editors, Cooperative Control, pages 229-239, New York, NY, 2005. Springer Lecture Notes in Control \& Information Sciences.

[24] H. K. Khalil. Nonlinear systems. Prentice-Hall, Upper Saddle River, NJ, second edition, 1995.

[25] C. Lanczos. The variational principles of mechanics. Dover, Mineola, NY, fourth edition, 1986.

[26] J. P. Hespanha and A. S. Morse. Stability of switched systems with average dwell-time. In Proceedings of the 38th IEEE Conference on Decision \& Control, pages 2655-2660, 1999.
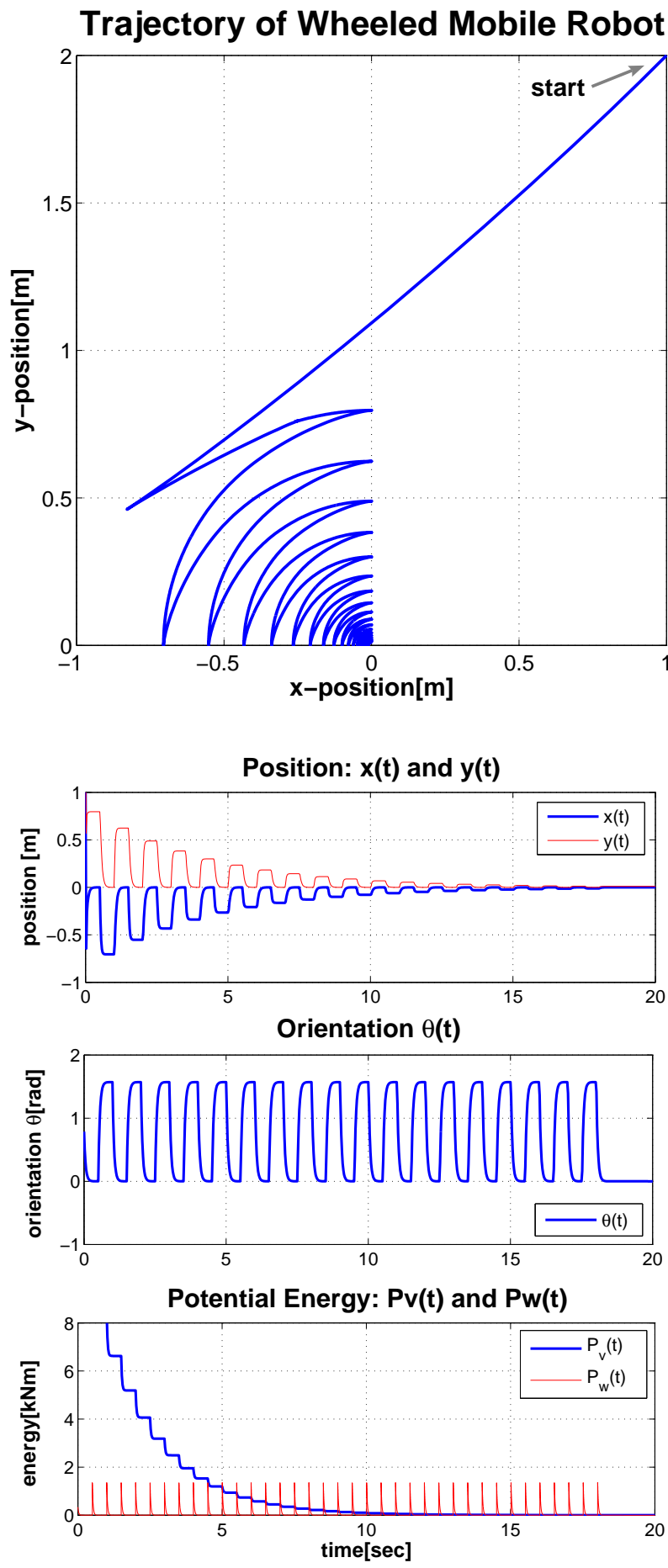

Fig. 4. First simulation results with $\theta_{1}=0$ and $\theta_{2}=\pi / 2$. 

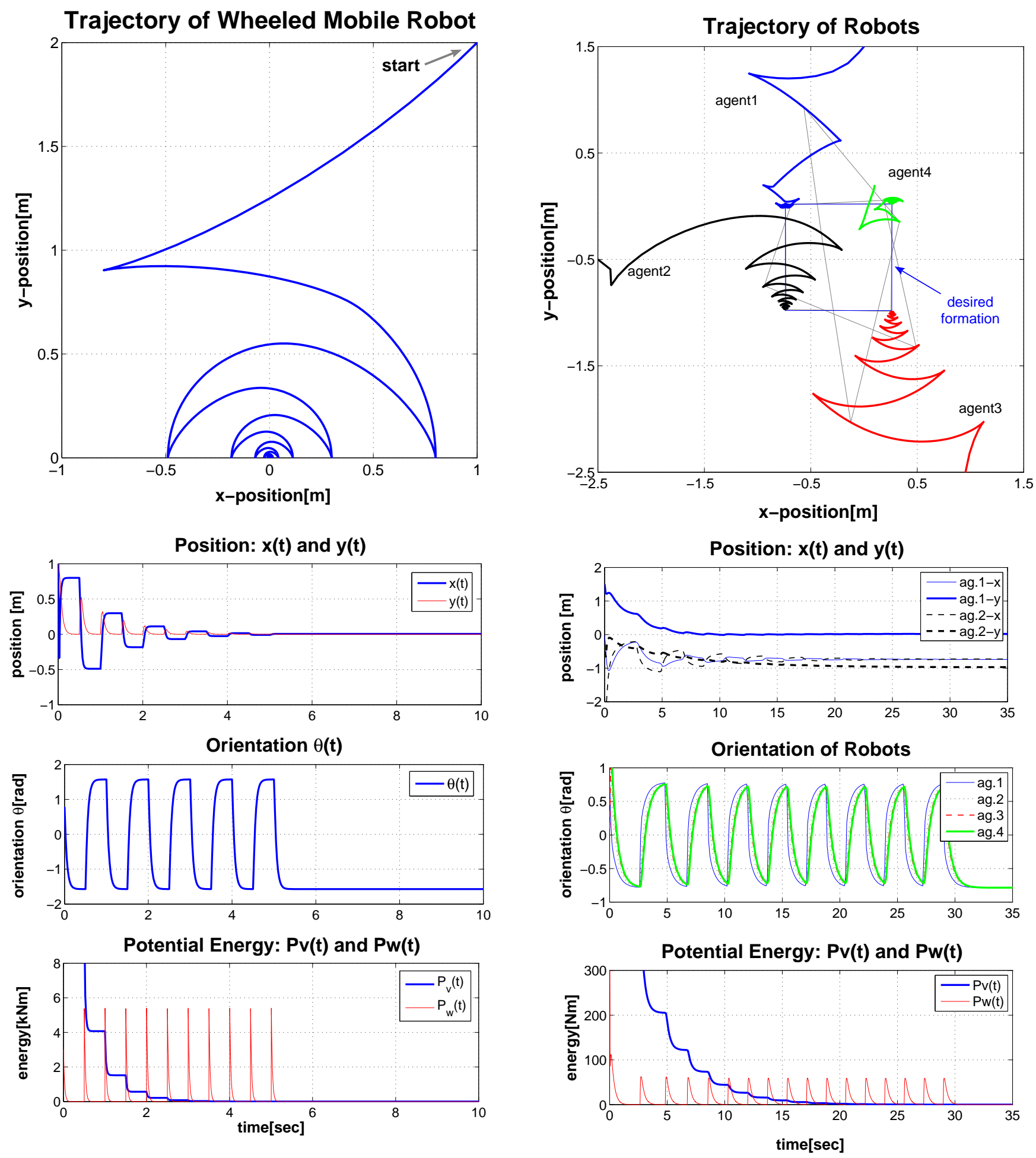

Fig. 5. Second simulation results with $\theta_{1}=-\pi / 2$ and $\theta_{2}=\pi / 2$.

Fig. 6. Coordination of multiple wheeled mobile robots. 